\title{
MARKARIAN GALAXIES IN THE VICINITY OF THE COMA CLUSTER
}

M. A. Arakelian

The distribution of Markarian galaxies in annular zones at different angular distances $\theta$ (degrees) from the centre of the Coma cluster is shown in the Table. The coordinates of the centre for the equinox

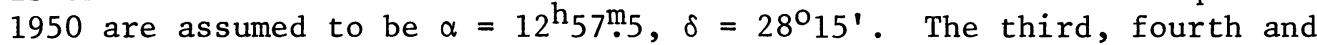
fifth columns of the Table contain the number of Markarian galaxies in each zone, the solid angle in square degrees and their mean surface densities respectively. It can be seen that an excess surface density of Markarian galaxies exists not only in the very central zone I but in the annular zone II as well. Indeed, the ratio $\mathrm{n} / \mathrm{\omega}$ in zone II is more than twice the value corresponding to the whole area surveyed (and to zones III and IV).

\begin{tabular}{|c|c|c|c|c|c|c|c|c|c|}
\hline Zone & & $\theta$ & $\mathrm{n}$ & $\omega$ & $\mathrm{n} / \mathrm{\omega}$ & $n^{\prime}$ & $\mathrm{n}^{\prime \prime}$ & $\langle\theta\rangle$ & $n^{\prime \prime} / n^{\prime}$ \\
\hline$I$ & 0 & -1.5 & 7 & 7 & 1.00 & 6 & 2 & 0.8 & 0.33 \\
\hline II & 1.5 & -6.0 & 22 & 106 & 0.21 & 15 & 10 & 4.8 & 0.67 \\
\hline III & 6.0 & -12.0 & 38 & 338 & 0.11 & 31 & 5 & 8.6 & 0.16 \\
\hline IV & & 12.0 & & & 0.10 & 570 & 71 & & 0.12 \\
\hline
\end{tabular}

The distribution of redshifts of Markarian galaxies indicates that there is an excess of these galaxies round the centre of the Coma cluster. The sixth column of the Table contains the numbers of galaxies with known redshifts and the seventh gives the numbers of galaxies with redshifts in the interval of $0.022-0.025$. The eighth column contains the mean $\theta$ of galaxies of the seventh column. One can see from the ninth column that the relative number of galaxies having redshifts between $0.024\left(1-\sin 6^{\circ}\right)$ and $0.024\left(1+\sin 6^{\circ}\right)$ in zone II is five times higher than that of the galaxies in zone IV $(0.024$ is the mean redshift of galaxies of the Coma cluster).

The luminosities of eight out of the above ten galaxies with redshifts close to 0.024 are higher than $\mathrm{M}_{\mathrm{p}}=20.0$ for $\mathrm{H}=50 \mathrm{~km} \mathrm{~s} \mathrm{~m}^{-1} \mathrm{Mpc}^{-1}$. Thus the density of luminous Markarian galaxies in the vicinity of the Coma cluster is nearly an order higher than their mean space density.

\section{DISCUSSION}

Huchra: My result for the Markarian luminosity function at the bright end agrees well with those of $\mathrm{Dr}$ Arakelian and I suggest that this may be due to an increased percentage of the Seyfert galaxies. 
Arakelian: Seyfert galaxies do not show a concentration towards the Coma cluster but the statistics are very small.

\section{REMARKS ON THE ANGULAR DISTRIBUTION OF MARKARIAN GALAXIES}

\section{Piotr Flin and Marek Urbanik}

Lists I-IX of Markarian galaxies have been used as the basic observational material. An attempt has been made to form a statistically complete sample. Two regions (region I $6.8<\alpha<14.2,41^{\circ}<\delta<77^{\circ}$, region II $6.8<\alpha<13$ h 8 , $5^{\circ}<\delta<32^{\circ}$ ) which appeared to be roughly complete have been carefully examined. After preliminary tests the first two hours in R.A. of the region II were removed from the analysis. The method of statistical reduction (F1in et al. 1974, A. Zieba 1975) and the maximum likelihood estimate of the number-magnitude counts (Crawford et a1. 1970) were applied as a combined test in their practical version (Urbanik 1976,1977). We have examined the whole region, as well as a number of smaller sub-regions varying in size and position. The angular sizes investigated ranged from $1^{\circ}$ up to $30^{\circ}$. Our analysis of 15 galaxy sets led to the following conclusions.

A much higher tendency toward clustering in region II in comparison with region I is accompanied by a significant decrease of surface density and flattening of number-magnitude counts. This effect varies across region II. This situation remains unchanged after restricting the analysis to galaxies brighter than $16^{\mathrm{m}}$. It has been found that multiple re-surveying of some small fields causes a sharp increase of density within them. These facts resemble the behaviour of radio source counts when investigations are carried out below the completeness limit of the radio survey. Therefore we infer that the completeness of the region II is considerably worse than that of the region I. Moreover the incompleteness is independent of the strength of the UV. The properties of the distribution of galaxies in region I do not differ qualitatively from those obtained for fainter normal galaxies (S. Zieba 1977). This similarity disappears in region II. In the light of these conclusions it seems that any large-scale statistical analysis of Markarian galaxies requires improvement in the completeness of the observational data.

\section{REFERENCES}

Crawford, D.F., Jauncey, D.L. and Murdoch, M.S., 1970. Astroph. J.,

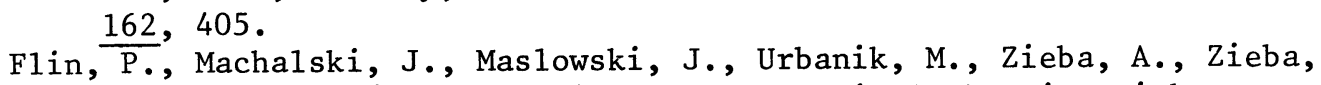
S., 1974. In "Confrontation of Cosmological Theories with Observational Data" (ed. M. S. Longair), p.121 (D. Reide1, Dordrecht).

Urbanik, M., 1976. Acta Cosmologica, 5, 97. Urbanik, M., 1977. Thesis, Jagiellonian University. Zieba, A., 1975. Acta Cosmologica, 3, 75. Zieba, S., 1977. Rozprawy habilitacyjne U.J., No.2, Krakow. 